DOI: 10.17516/1997-1397-2020-13-5-533-546

УДК 517.956.223

\title{
Nonlocal Problem for a Three-dimensional Elliptic Equation with Singular Coefficients in a Rectangular Parallelepiped
}

\author{
Kamoliddin T. Karimov* \\ Ferghana State University \\ Ferghana, Uzbekistan
}

Received 20.05.2020, received in revised form 14.06.2020, accepted 08.07.2020

\begin{abstract}
The nonlocal problem for an elliptic equation with two singular coefficients in a rectangular parallelepiped is studied. The uniqueness of the solution of the problem is proved with the use of the method of energy integrals. The spectral Fourier method based on the separation of variables is used to prove the existence of solutions. The solution of the problem is constructed as double Fourier series in terms of a sum of trigonometric and Bessel functions. Under some conditions on parameters and given functions the uniform convergence of the constructed series and its derivatives up to the second order inclusive is proved.
\end{abstract}

Keywords: elliptic type equation, nonlocal problem, singular coefficient, spectral method, parallelepiped.

Citation: K.T.Karimov, Nonlocal Problem for a Three-dimensional Elliptic Equation with Singular Coefficients in a Rectangular Parallelepiped, J. Sib. Fed. Univ. Math. Phys., 2020, 13(5), 533-546.

DOI: 10.17516/1997-1397-2020-13-5-533-546.

\section{Introduction. Formulation of the problem}

Let us consider a three-dimensional elliptic equation with two singular coefficients

$$
L u \equiv u_{x x}+u_{y y}+u_{z z}+\frac{2 \beta}{y} u_{y}+\frac{2 \gamma}{z} u_{z}=0,
$$

in a rectangular parallelepiped $\Omega=\{(x, y, z): 0<x<a, 0<y<b, 0<z<c\}$, where $\beta$ and $\gamma$ are real numbers with $\beta, \gamma<1 / 2 ; u=u(x, y, z)$ is an unknown function.

Consider the following problem and study its unique solvability.

Dezin's problem. Find a function $u(x, y, z) \in C(\bar{\Omega}) \cap C^{1}(\bar{\Omega} \cap(\{x=0\} \cup\{x=a\})) \cap C^{2}(\Omega)$ that satisfies equation (1) in the domain $\Omega$ and the following conditions

$$
\begin{gathered}
u(0, y, z)=u(a, y, z), \quad u_{x}(0, y, z)=u_{x}(a, y, z), \quad 0 \leqslant y \leqslant b, 0 \leqslant z \leqslant c, \\
u(x, y, 0)=0, \quad u(x, y, c)=0, \quad 0 \leqslant x \leqslant a, \quad 0 \leqslant y \leqslant b, \\
u(x, 0, z)=f_{1}(x, z), \quad 0 \leqslant x \leqslant a, \quad 0 \leqslant z \leqslant c, \\
u(x, b, z)=f_{2}(x, z), \quad 0 \leqslant x \leqslant a, \quad 0 \leqslant z \leqslant c,
\end{gathered}
$$

where $f_{1}(x, z)$ and $f_{2}(x, z)$ are given continuous functions.

*karimovk80@mail.ru http://orcid.org/0000-0002-9098-4116

(c) Siberian Federal University. All rights reserved 
Desin studied equation

$$
(d / d t) u-A u=f, 0 \leqslant t \leqslant a
$$

with the boundary condition $\left.b u\right|_{t=0}-\left.u\right|_{t=a}=g[1]$. Here, it is assumed that function $u(t)$ takes values in a complex Banach space $B$ when $t \in[0, a], A: B \rightarrow B$ is an unbounded linear operator commuting with $d / d t$ and a density domain of definition, and $b$ is a complex number. It is also explained that the given conditions are "nonlocal" in the sense that they determine the relationship between the values of the unknown function at different points of the boundary.

Nonlocal boundary value problems are very interesting problems. They generalize the classical problems and, at the same time, they are naturally obtained when constructing mathematical models of real processes and phenomena in physics, engineering, etc (for an extended discussion see [2-6]). Problems with nonlocal conditions for partial differential equations have been studied by many authors. Below an overview of problems close to the Dezin problem is given. They are formulated and studied in two-dimensional domains.

Frankl [7] considered the flow around a finite symmetric profile by a subsonic velocity stream and formulated the problem for the Chaplygin equation in a mixed domain with a nonlocal condition of the form $u(0, y)=u(0,-y)$. In addition, the local condition $u_{x}(0, y)=0$ was fulfilled. Ionkin [8] proved the existence of a solution to a nonlocal problem with conditions $u_{x}(0, y)=u_{x}(1, y), u(0, y)=0,0 \leqslant y \leqslant T$ and $u(x, 0)=\tau(x), 0 \leqslant x \leqslant 1$ for the heat equation using the spectral analysis method. The uniqueness of the solution of this problem was proved [9]. Such conditions are encountered, for example, in problems of particle diffusion in turbulent plasma and in problems of heat propagation in a thin heated rod if the law of change of the total amount of heat of the rod is given. Ionkin and Moiseev [10] proved the unique solvability of the problem for the heat equation with conditions

$$
\begin{aligned}
& a_{1} u_{x}(0, t)+b_{1} u_{x}(1, t)+a_{0} u(0, t)+b_{0} u(1, t)=0, \\
& c_{1} u_{x}(0, t)+d_{1} u_{x}(1, t)+c_{0} u(0, t)+d_{0} u(1, t)=0,
\end{aligned}
$$

where $a_{j}, b_{j}, c_{j}, d_{j}, j=\overline{0,1}$ are given constants.

Lerner and Repin [11] studied the following problem in half-strip $D=$ $\{(x, y): 0<x<1, y>0\}$. Find a function $u(x, y)$ with properties

$$
\begin{gathered}
u(x, y) \in C(\bar{D}) \cap C^{1}(D \cup\{x=0\}) \cap C^{2}(D) ; \\
y^{m} u_{x x}+u_{y y}=0, \quad(x, y) \in D, \quad m>-1 ; \\
u(x, y) \rightarrow 0 \text { at } y \rightarrow+\infty \text { uniformly in } x \in[0,1] ; \\
u(0, y)-u(1, y)=\varphi_{1}(y), \quad u_{x}(0, y)=\varphi_{2}(y), \quad y \geqslant 0 ; \quad u(x, 0)=\tau(x), 0 \leqslant x \leqslant 1,
\end{gathered}
$$

where $\tau(x), \varphi_{1}(y)$ and $\varphi_{2}(y)$ are given sufficiently smooth functions, and $\tau(x)$ is orthogonal to the system of functions $1, \cos (2 n+1) \pi x, n=0,1,2, \ldots$. The similar problem was studied in the half-strip $D$ for equation [12]

$$
u_{x x}+u_{y y}+\frac{2 p}{y} u_{y}-b^{2} u=0, p, b \in R,
$$

Assuming that $\varphi_{1}(y) \equiv 0$ and $\varphi_{2}(y) \equiv 0$. The uniqueness of the solution of this problem is proved on the basis of the extremum principle. Using the methods of separation of variables and integral transforms, the solvability of the problem in question was established. Moiseev [13] 
studied the following nonlocal boundary-value problem in the half-strip $D$ for degenerate elliptic equation of the form

$$
\begin{gathered}
y^{m} u_{x x}+u_{y y}=0, \quad m>-2 ; \\
u(x, 0)=f(x), \quad 0 \leqslant x \leqslant 1 ; \quad u(0, y)=u(1, y), \quad u_{x}(0, y)=0, y \geqslant 0 ; \\
f(x) \in C^{2+\alpha}[0,1], f(0)=f(1), f^{\prime}(0)=0 .
\end{gathered}
$$

Using the spectral analysis method, the uniqueness and existence of the solution of this problem were proved in the class of functions $u(x, y) \in C(\bar{D}) \cap C^{2}(D)$. Functions tend to zero or are bounded at infinity. Moreover, the solution of the problem was constructed in the form of the sum of the biorthogonal series. These results are also applicable to equations $y^{m} u_{x x}+u_{y y}-b^{2} y^{m} u=0, m, b \in R$, with $b \geqslant 0, m>0$ [14]. Equation $y^{m} u_{x x}--u_{y y}-b^{2} y^{m} u=0$ was studied in rectangular domain $\{(x, y): 0<x<1,0<y<T\}$ [15], where $m>0, b \geqslant 0$, $T>0$ are given real numbers. Initial conditions $u(x, 0)=\tau(x), u_{y}(x, 0)=\nu(x), 0 \leqslant x \leqslant 1$ and nonlocal boundary conditions $u(0, y)=u(1, y), u_{x}(0, y)=0$ or $u_{x}(0, y)=u_{x}(1, y), u(1, y)=0$ at $0 \leqslant y \leqslant T$ were assumed. The uniqueness and existence theorems are proved with the use of the spectral analysis method. Equation

$$
u_{x x}+\operatorname{sgn} y u_{y y}+\frac{2 p}{|y|} u_{y}+k u=0, \quad p \geqslant 1 / 2, \quad k \in R
$$

was considered in domain $D=\{(x, y): 0<x<1, y<\alpha\}, \alpha>0[16]$ and the following problem was studied

$$
\begin{gathered}
u \in C(\bar{D}) \cap C^{2}(D \backslash\{y=0\}), \quad L u=0 ; \\
u(0, y)=u(1, y), \quad u_{x}(0, y)=0, y<\alpha ; \quad u(x, \alpha)=\varphi(x), 0<x<1,
\end{gathered}
$$

where $\varphi(x)$ is the given continuous function that satisfies condition $\varphi(0)=\varphi(1)$.

Nonlocal problems for inhomogeneous Lavrentev-Bitsadze equation and for equation of mixed elliptic-hyperbolic type with power degeneration were studied in the rectangular domains [17-21].

However, nonlocal problems for equations with singular coefficients in three-dimensional domains remain poorly understood.

\section{Construction of eigenfunctions}

To find a solution to the Dezin problem we apply the Fourier method [22]. Let us find nontrivial solutions of problem (1)-(3). Using separation of variables $u(x, y, z)==W(x, z) Q(y)$, we obtain from equation (1)

$$
\begin{gathered}
Q^{\prime \prime}(y)+\frac{2 \beta}{y} Q^{\prime}(y)-\lambda Q(y)=0, \quad 0<y<b, \\
W_{x x}+W_{z z}+W_{x}+\frac{2 \gamma}{z} W_{z}+\lambda W=0, \quad 0<x<a, \quad 0<z<c,
\end{gathered}
$$

where $\lambda \in R$ is the separation constant.

Taking into account conditions (2) and (3), we obtain for equation (7) the following eigenvalue problem in the domain $\Pi=\{(x, z): 0<x<a, 0<z<c\}$ : find the values of parameter $\lambda$ and the corresponding nontrivial solutions $W(x, z) \in C(\bar{\Pi}) \cap C^{1}(\bar{\Pi} \cap(\{x=0\} \cup\{x=a\})) \cap C^{2}(\Omega)$ of equation (7) in $\bar{\Pi}$ that satisfy conditions $W(0, z)=W(a, z), 0 \leqslant z \leqslant c ; W(x, 0)=0$, $W(x, c)=0,0 \leqslant x \leqslant a$. 
By separating variables $W(x, z)=X(x) Z(z)$, this problem reduces to the following eigenvalue problem for the ordinary differential equation:

$$
\begin{gathered}
L_{\lambda-\mu}^{\gamma} Z(z)=Z^{\prime \prime}(z)+(2 \gamma / z) Z^{\prime}(z)+(\lambda-\mu) Z(z)=0, \quad Z(0)=0, \quad Z(c)=0 \\
L_{\mu}^{0} X(x)=0, \quad X(0)=X(a), \quad X^{\prime}(0)=X^{\prime}(a),
\end{gathered}
$$

where $\mu \in R$ is the separation constant.

Let us find first a solution of problem (9). It is easy to verify that for $\mu<0$ problem (9) has only trivial solutions. At $\mu=0$ the solution of problem (9) is $X(x)=d_{0}\left(d_{0} \neq 0\right.$ is some constant). Consider now the case $\mu>0$. Since boundary conditions in problem (9) are periodic conditions, the problem is regular. In addition, it is easy to verify that boundary-value problem (9) is a self-adjoint problem [23].

Substituting the general solution of equation $L_{\mu}^{0} X(x)=0$

$$
X(x)=d_{1} \sin \sqrt{\mu} x+d_{2} \cos \sqrt{\mu} x
$$

into nonlocal conditions $X(0)=X(a)$ and $X^{\prime}(0)=X^{\prime}(a)$, we obtain the following system of equations

$$
\left\{\begin{array}{l}
d_{1} \sin \sqrt{\mu} a+d_{2}(\cos \sqrt{\mu} a-1)=0, \\
d_{1}(\cos \sqrt{\mu} a-1)-d_{2} \sin \sqrt{\mu} a=0 .
\end{array}\right.
$$

Setting the main determinant of this system to zero, we find $\cos \sqrt{\mu} a-1=0$. Solving this equation, we find the eigenvalues of the problem: $\mu_{n}=(2 \pi n / a)^{2}, n \in N$. Let us substitute $\mu=\mu_{n}$ into (10) and (11). It is easy to verify that the eigenvalue $\mu_{0}$ is simple, and it corresponds to one normalized eigenfunction $1 / \sqrt{a}$. Eigenvalues $\mu_{n}, n \in N$ have multiplicity 2 and they correspond to two normalized eigenfunctions $\sqrt{2 / a} \cos (2 \pi n x / a), \sqrt{2 / a} \sin (2 \pi n x / a)$. Therefore, the eigenvalues and the eigenfunctions of problem (9) that correspond to these eigenvalues can be represented in the form of $\mu_{n}=(2 \pi n / a)^{2}, n \in N \cup\{0\}$ and

$$
X_{0}(x)=\frac{1}{\sqrt{a}}, \quad X_{2 n-1}(x)=\sqrt{\frac{2}{a}} \sin \left(\frac{2 \pi n x}{a}\right), \quad X_{2 n}(x)=\sqrt{\frac{2}{a}} \cos \left(\frac{2 \pi n x}{a}\right), n \in N .
$$

The system of eigenfunctions (12) is orthogonal and complete in the space $L_{2}[0, a]$, and it forms orthonormal basis in it (see, for example, [20,27]).

Now we turn to the study of problem (8). We consider equation $L_{\lambda-\mu}^{\gamma} Z(z)=0$ and find its general solution at $\mu=\mu_{n}$. Introducing $Z(z)=\left(t / \sqrt{\lambda-\mu_{n}}\right)^{1 / 2-\gamma} p(t)$, where $t=\sqrt{\lambda-\mu_{n}} z$, $\lambda>\mu_{n}$ (at $\lambda \leqslant \mu_{n}$, problem (8) has only trivial solutions), from equation $L_{\lambda-\mu_{n}}^{\gamma} Z(z)=0$ we obtain the Bessel equation [24]:

$$
t^{2} p^{\prime \prime}(t)+t p^{\prime}(t)+\left[t^{2}-(1 / 2-\gamma)^{2}\right] p(t)=0
$$

Taking into account the form of the general solution of the last equation [24] and the introduced notation, we obtain the general solution of equation $L_{\lambda-\mu_{n}}^{\gamma} Z(z)=0$ in the form

$$
Z_{n}(z)=d_{1 n} z^{1 / 2-\gamma} J_{1 / 2-\gamma}\left(\sqrt{\lambda-\mu_{n}} z\right)+d_{2 n} z^{1 / 2-\gamma} Y_{1 / 2-\gamma}\left(\sqrt{\lambda-\mu_{n}} z\right),
$$

where $d_{1}, d_{2}$ are arbitrary constants, $J_{l}(z)$ and $Y_{l}(z)$ are the Bessel functions of the first and second kind of the order $l$, respectively [24]. 
It follows from (13) that a solution of equation $L_{\lambda-\mu_{n}}^{\gamma} Z(z)=0$ that satisfies condition $Z(0)=0$ is $Z_{n}(z)=d_{1} z^{1 / 2-\gamma} J_{1 / 2-\gamma}\left(\sqrt{\lambda-\mu_{n}} z\right)$. Substituting this into condition $Z(c)=0$, we obtain the condition for the existence of a nontrivial solution of problem (8):

$$
J_{1 / 2-\gamma}\left(\sqrt{\lambda-\mu_{n}} c\right)=0, \quad n \in N
$$

It is known that when $l>-1$ the Bessel function $J_{l}(z)$ has a countable number of zeros. They are real and has pairwise opposite signs [24]. Since $1 / 2-\gamma>0$ then equation (14) has a countable number of real roots. Denoting the mth positive root of equation (14) at $n=k$ by $\delta_{k m}$, we have values of parameter $\lambda$ for which there are nontrivial solutions of problem (8): $\lambda_{n m}=\mu_{n}+\left(\delta_{n m} / c\right)^{2}, n=0,1,2, \ldots, m \in N$.

Assuming in (13) that $\lambda=\lambda_{n m}, d_{1 n}=1, d_{2 n}=0, n=0,1,2, \ldots, m \in N$, we obtain nontrivial solutions (eigenfunctions) of problem (8) up to a constant factor

$$
Z_{n m}(z)=z^{1 / 2-\gamma} J_{1 / 2-\gamma}\left(\delta_{n m} z / c\right), \quad n=0,1,2, \ldots, m \in N .
$$

According to [24], for each $n$ the system of eigenfunctions (15) is complete in space $L_{2}[0, c]$ with the weight $z^{2 \gamma}$.

Assuming in equation (6) $\lambda=\lambda_{n m}$, we find its general solution

$$
Q_{n m}(y)=a_{n m} y^{1 / 2-\beta} I_{1 / 2-\beta}\left(\sqrt{\lambda_{n m}} y\right)+b_{n m} y^{1 / 2-\beta} K_{1 / 2-\beta}\left(\sqrt{\lambda_{n m}} y\right), 0 \leqslant y \leqslant b,
$$

here $a_{n m}$ and $b_{n m}$ are arbitrary constants, $I_{l}(y)$ and $K_{l}(y)$ are the modified Bessel functions of the first and third kind of the order $l$, respectively [24].

\section{The uniqueness of the solution}

The proof of the uniqueness of the solution of the Dezin problem is based on the lemma given below.

Lemma 1. If $\beta, \gamma<1 / 2$, function $u(x, y, z)$ is the solution of equation (1), and it satisfies conditions $u(x, 0, z)=0$ and $u(x, y, 0)=0$ then inequalities $\left|\lim _{y \rightarrow 0} y^{2 \beta} u_{y}(x, y, z)\right|<+\infty$ and $\left|\lim _{z \rightarrow 0} z^{2 \gamma} u_{z}(x, y, z)\right|<+\infty$ are satisfied.

Proof. Separating variables by the formula $u(x, y, z)=X(x) Q(y) Z(z)$, from equation (1) with the variables $y$ and $z$ we obtain the ordinary differential equations (6) and $L_{\lambda-\mu}^{\gamma} Z(z)=0$. Using the general solutions of these equations, it is easy to verify that the solutions of equations (6) and $L_{\lambda-\mu}^{\gamma} Z(z)=0$ that satisfy conditions $Q(0)=0$ and $Z(0)=0$ respectively at $\beta, \gamma<1 / 2$ have the form (up to a constant factor) $Q(y)=y^{1 / 2-\beta} I_{1 / 2-\beta}(\sqrt{\lambda} y)$ and $Z(z)=z^{1 / 2-\gamma} J_{1 / 2-\gamma}(\sqrt{\lambda-\mu} z)$. Taking the first-order derivative of these functions using relations [24]

$$
\frac{d}{d x}\left[x^{ \pm \nu} J_{\nu}(x)\right]= \pm x^{ \pm \nu} J_{\nu \mp 1}(x), \quad \frac{d}{d x}\left[x^{ \pm \nu} I_{\nu}(x)\right]=x^{ \pm \nu} J_{\nu \mp 1}(x),
$$

we have $Q^{\prime}(y)=\sqrt{\lambda} y^{1 / 2-\beta} I_{-1 / 2-\beta}(\sqrt{\lambda} y), Z^{\prime}(z)=\sqrt{\lambda-\mu} z^{1 / 2-\gamma} J_{-1 / 2-\gamma}(\sqrt{\lambda-\mu} z)$. It follows that $\left|\lim _{y \rightarrow 0} y^{2 \beta} Q^{\prime}(y)\right|<+\infty,\left|\lim _{z \rightarrow 0} z^{2 \gamma} Z^{\prime}(z)\right|<+\infty$. Taking into account that $u(x, y, z)=$ 
$=X(x) Q(y) Z(z)$, we have that $\left|\lim _{y \rightarrow 0} y^{2 \beta} u_{y}(x, y, z)\right|<+\infty$ and $\left|\lim _{z \rightarrow 0} z^{2 \gamma} u_{z}(x, y, z)\right|<+\infty$. Lemma 1 is proved.

Now we turn to the proof of the uniqueness of the solution of the Dezin problem.

Theorem 1. The Dezin problem does not have more than one solution.

Proof. Let $V_{1}(x, y, z)$ and $V_{2}(x, y, z)$ be solutions of the Dezin problem. Then function $u(x, y, z)=$ $V_{1}(x, y, z)-V_{2}(x, y, z)$ satisfies equation (1), conditions (2), (3) and homogeneous boundary conditions corresponding to (4) and (5). We prove that $u(x, y, z) \equiv 0$ in $\bar{\Omega}$.

In domain $\Omega$ we have the identity

$$
y^{2 \beta} z^{2 \gamma} u L u=\left(y^{2 \beta} z^{2 \gamma} u u_{x}\right)_{x}+\left(y^{2 \beta} z^{2 \gamma} u u_{y}\right)_{y}+\left(y^{2 \beta} z^{2 \gamma} u u_{z}\right)_{z}-y^{2 \beta} z^{2 \gamma}\left(u_{x}^{2}+u_{y}^{2}+u_{z}^{2}\right)=0 .
$$

Integrating this identity over domain

$$
\Omega_{\varepsilon_{1} \varepsilon_{2} \varepsilon_{3} \varepsilon_{4}}^{\varepsilon_{5} \varepsilon_{6}}=\left\{(x, y, z): \varepsilon_{1}<x<a-\varepsilon_{2}, \varepsilon_{3}<y<b-\varepsilon_{4}, \varepsilon_{5}<z<c-\varepsilon_{6}\right\},
$$

where $\varepsilon_{j}, j=\overline{1,6}$ are sufficiently small positive numbers, we have

$$
\begin{aligned}
\iiint_{\Omega_{5} \varepsilon_{6} \varepsilon_{3} \varepsilon_{4}}\left[\left(y^{2 \beta} z^{2 \gamma} u u_{x}\right)_{x}+\left(y^{2 \beta} z^{2 \gamma} u u_{y}\right)_{y}+\right. & \left.\left(y^{2 \beta} z^{2 \gamma} u u_{z}\right)_{z}\right] d x d y d z= \\
& =\iiint_{\substack{\varepsilon_{\varepsilon_{1}} \varepsilon_{5} \varepsilon_{6} \\
\Omega_{\varepsilon_{1} \varepsilon_{2} \varepsilon_{3} \varepsilon_{4}}}}\left[y^{2 \beta} z^{2 \gamma}\left(u_{x}^{2}+u_{y}^{2}+u_{z}^{2}\right)\right] d x d y d z .
\end{aligned}
$$

Obviously, if $\varepsilon_{j}, j=\overline{1,6}$ tends to zero then $\Omega_{\varepsilon_{1} \varepsilon_{2} \varepsilon_{3} \varepsilon_{4}}^{\varepsilon_{5} \varepsilon_{6}} \rightarrow \Omega$.

Applying the Gauss-Ostrogradsky formula [22] to the left hand side of equality (18), we obtain after some transformations that

$$
\begin{gathered}
\int_{\varepsilon_{5}}^{c-\varepsilon_{6}} \int_{\varepsilon_{3}}^{b-\varepsilon_{4}} y^{2 \beta} z^{2 \gamma}\left[u_{x}\left(a-\varepsilon_{2}, y, z\right)-u_{x}\left(\varepsilon_{1}, y, z\right)\right] u\left(a-\varepsilon_{2}, y, z\right) d y d z+ \\
+\int_{\varepsilon_{5}}^{c-\varepsilon_{6}} \int_{\varepsilon_{3}}^{b-\varepsilon_{4}} y^{2 \beta} z^{2 \gamma}\left[u\left(a-\varepsilon_{2}, y, z\right)-u\left(\varepsilon_{1}, y, z\right)\right] u_{x}\left(\varepsilon_{1}, y, z\right) d y d z+ \\
+\int_{\varepsilon_{5}}^{c-\varepsilon_{6}} \int_{\varepsilon_{1}}^{a-\varepsilon_{2}} z^{2 \gamma}\left[\left(b-\varepsilon_{4}\right)^{2 \beta} u\left(x, b-\varepsilon_{4}, z\right) u_{y}\left(x, b-\varepsilon_{4}, z\right)-\varepsilon_{3}^{2 \beta} u\left(x, \varepsilon_{3}, z\right) u_{y}\left(x, \varepsilon_{3}, z\right)\right] d x d z+ \\
+\int_{\varepsilon_{3}}^{b-\varepsilon_{4}} \int_{\varepsilon_{1}}^{a-\varepsilon_{2}} y^{2 \beta}\left[\left(c-\varepsilon_{6}\right)^{2 \gamma} u\left(x, y, c-\varepsilon_{6}\right) u_{z}\left(x, y, c-\varepsilon_{6}\right)-\varepsilon_{5}^{2 \gamma} u\left(x, y, \varepsilon_{5}\right) u_{z}\left(x, y, \varepsilon_{5}\right)\right] d x d y= \\
=\iiint_{\Omega_{\varepsilon_{5} \varepsilon_{2} \varepsilon_{3} \varepsilon_{4}}}\left[y^{2 \beta} z^{2 \gamma}\left(u_{x}^{2}+u_{y}^{2}+u_{z}^{2}\right)\right] d x d y d z .
\end{gathered}
$$

Hence, taking the limit at $\varepsilon_{j} \rightarrow 0, j=\overline{1,6}$ and taking into account the conditions of Lemma $1,\left|u_{y}(x, b, z)\right|<+\infty,\left|u_{z}(x, y, c)\right|<+\infty$ and homogeneous boundary conditions, we obtain

$$
\iiint_{\Omega}\left[y^{2 \beta} z^{2 \gamma}\left(u_{x}^{2}+u_{y}^{2}+u_{z}^{2}\right)\right] d x d y d z=0 .
$$


Therefore $u_{x}(x, y, z) \equiv u_{y}(x, y, z) \equiv u_{z}(x, y, z) \equiv 0,(x, y, z) \in \Omega$. Then $u(x, y, z) \equiv$ const, $(x, y, z) \in \Omega$. Since $u(x, y, z) \in C(\bar{\Omega})$ and $u(x, 0, z) \equiv 0$ then $u(x, y, z) \equiv 0,(x, y, z) \in \bar{\Omega}$. The theorem is proved.

\section{Construction and justification of the solution of the Dezin problem}

Let us assume that solution of the Dezin problem in domain $\Omega$ has the form

$$
\begin{aligned}
& u(x, y, z)=\sum_{n=0}^{\infty} \sum_{m=1}^{\infty} X_{n}(x) Q_{n m}(y) Z_{n m}(z)= \\
&=\frac{1}{\sqrt{a}} \sum_{m=1}^{\infty} z^{1 / 2-\gamma} J_{1 / 2-\gamma}\left(\frac{\delta_{0 m} z}{c}\right) y^{1 / 2-\beta}\left[a_{0 m} I_{1 / 2-\beta}\left(\delta_{0 m} y\right)+b_{0 m} K_{1 / 2-\beta}\left(\delta_{0 m} y\right)\right]+ \\
& \quad+\sqrt{\frac{2}{a} \sum_{n=1}^{\infty} \sum_{m=1}^{\infty}}\left(\sin \frac{2 \pi n x}{a}+\cos \frac{2 \pi n x}{a}\right) z^{1 / 2-\gamma} J_{1 / 2-\gamma}\left(\frac{\delta_{n m} z}{c}\right) \times \\
& \quad \times y^{1 / 2-\beta}\left[a_{n m} I_{1 / 2-\beta}\left(\sqrt{\lambda_{n m}} y\right)+b_{n m} K_{1 / 2-\beta}\left(\sqrt{\lambda_{n m}} y\right)\right] .
\end{aligned}
$$

Each term of series (19) satisfies equation (1) and conditions (2) and (3). Assuming that this series converges absolutely and uniformly, we find constants $a_{n m}$ and $b_{n m}$ from the requirement that function (19) must satisfy boundary conditions (4) and (5). First, substituting it in conditions (4), we obtain

$$
\frac{F_{0}(z)}{\sqrt{a}}+\sqrt{\frac{2}{a}} \sum_{n=1}^{\infty} F_{n}(z)\left(\sin \frac{2 \pi n x}{a}+\cos \frac{2 \pi n x}{a}\right)=z^{\gamma-1 / 2} f_{1}(x, z),
$$

where

$$
F_{n}(z)=2^{-1 / 2-\beta} \Gamma(1 / 2-\beta) \sum_{m=1}^{\infty} J_{1 / 2-\gamma}\left(\frac{\delta_{n m} z}{c}\right)\left(\sqrt{\lambda_{n m}}\right)^{\beta-1 / 2} b_{n m} .
$$

Series $(20)$ and $(21)$ are called the Fourier series of functions $z^{\gamma-1 / 2} f_{1}(x, z)$ and $F_{n}(z)$ expanded in the system of the trigonometric and Bessel functions, respectively. The Fourier coefficients are determined from (20) as follows

$$
\begin{gathered}
F_{0}(z)=\frac{1}{\sqrt{a}} \int_{0}^{a} z^{\gamma-1 / 2} f_{1}(x, z) d x \\
F_{n}(z)=\frac{1}{\sqrt{2 a}} \int_{0}^{a}\left(\sin \frac{2 \pi n x}{a}+\cos \frac{2 \pi n x}{a}\right) z^{\gamma-1 / 2} f_{1}(x, z) d x .
\end{gathered}
$$

Since $F_{0}(z)$ and $F_{n}(z)$ are known we substitute them into (21), and unequivocally find coefficients $b_{0 m}$ and $b_{n m}$ :

$$
\begin{aligned}
b_{0 m} & =\frac{2^{3 / 2+\beta} \delta_{0 m}^{1 / 2-\beta}}{\sqrt{a}\left[c J_{3 / 2-\gamma}\left(\delta_{0 m}\right)\right]^{2} \Gamma(1 / 2-\beta)} f_{0 m}, \\
b_{n m} & =\frac{2^{1+\beta}\left(\sqrt{\lambda_{n m}}\right)^{1 / 2-\beta}}{\sqrt{a}\left[c J_{3 / 2-\gamma}\left(\delta_{n m}\right)\right]^{2} \Gamma(1 / 2-\beta)} f_{n m},
\end{aligned}
$$


where

$$
f_{n m}=\int_{0}^{c} \int_{0}^{a}\left(\sin \frac{2 \pi n x}{a}+\cos \frac{2 \pi n x}{a}\right) z^{1 / 2+\gamma} J_{1 / 2-\gamma}\left(\frac{\delta_{n m} z}{c}\right) f_{1}(x, z) d x d z, n+1, \quad m \in N .
$$

Now, substituting function (19) into condition (5), we have

$$
\frac{G_{0}(z)}{\sqrt{a}}+\sqrt{\frac{2}{a}} \sum_{n=1}^{\infty} G_{n}(z)\left(\sin \frac{2 \pi n x}{a}+\cos \frac{2 \pi n x}{a}\right)=z^{\gamma-1 / 2} b^{\beta-1 / 2} f_{2}(x, z),
$$

where

$$
G_{n}(z)=\sum_{m=1}^{\infty} J_{1 / 2-\gamma}\left(\frac{\delta_{n m} z}{c}\right)\left[a_{n m} I_{1 / 2-\beta}\left(\sqrt{\lambda_{n m}} b\right)+b_{n m} K_{1 / 2-\beta}\left(\sqrt{\lambda_{n m}} b\right)\right] .
$$

From relation (24) we find

$$
\begin{gathered}
G_{0}(z)=\frac{1}{\sqrt{a}} \int_{0}^{a} z^{\gamma-1 / 2} b^{\beta-1 / 2} f_{2}(x, z) d x \\
G_{n}(z)=\frac{1}{\sqrt{2 a}} \int_{0}^{a}\left(\sin \frac{2 \pi n x}{a}+\cos \frac{2 \pi n x}{a}\right) z^{\gamma-1 / 2} b^{\beta-1 / 2} f_{2}(x, z) d x .
\end{gathered}
$$

Substituting $G_{0}(z)$ and $G_{n}(z)$ into (25), we obtain

$$
\begin{gathered}
a_{0 m} I_{1 / 2-\beta}\left(\delta_{0 m} b\right)+b_{0 m} K_{1 / 2-\beta}\left(\delta_{0 m} b\right)=\frac{2 b^{\beta-1 / 2}}{\sqrt{a}\left[c J_{3 / 2-\gamma}\left(\delta_{0 m}\right)\right]^{2}} g_{0 m}, \\
a_{n m} I_{1 / 2-\beta}\left(\sqrt{\lambda_{n m}} b\right)+b_{n m} K_{1 / 2-\beta}\left(\sqrt{\lambda_{n m}} b\right)=\frac{\sqrt{2} b^{\beta-1 / 2}}{\sqrt{a}\left[c J_{3 / 2-\gamma}\left(\delta_{n m}\right)\right]^{2}} g_{n m},
\end{gathered}
$$

where

$$
g_{n m}=\int_{0}^{c} \int_{0}^{a}\left(\sin \frac{2 \pi n x}{a}+\cos \frac{2 \pi n x}{a}\right) z^{1 / 2+\gamma} J_{1 / 2-\gamma}\left(\frac{\delta_{n m} z}{c}\right) f_{2}(x, z) d x d z, \quad n+1, m \in N .
$$

Since $b_{0 m}$ and $b_{n m}$ are known then from the last system of equations we unequivocally find coefficients $a_{0 m}$ and $a_{n m}$ :

$$
\begin{gathered}
a_{0 m}=\frac{2 b^{\beta-1 / 2} g_{0 m}}{\sqrt{a}\left[c J_{3 / 2-\gamma}\left(\delta_{0 m}\right)\right]^{2} I_{1 / 2-\beta}\left(\delta_{0 m} b\right)}-\frac{2^{3 / 2+\beta} \delta_{0 m}^{1 / 2-\beta} K_{1 / 2-\beta}\left(\delta_{0 m} b\right) f_{0 m}}{\sqrt{a}\left[c J_{3 / 2-\gamma}\left(\delta_{0 m}\right)\right]^{2} \Gamma(1 / 2-\beta) I_{1 / 2-\beta}\left(\delta_{0 m} b\right)}, \\
a_{n m}=\frac{\sqrt{2} b^{\beta-1 / 2} g_{n m}}{\sqrt{a}\left[c J_{3 / 2-\gamma}\left(\delta_{n m}\right)\right]^{2} I_{1 / 2-\beta}\left(\sqrt{\lambda_{n m}} b\right)}-\frac{2^{1+\beta}\left(\sqrt{\lambda_{n m}}\right)^{1 / 2-\beta} K_{1 / 2-\beta}\left(\sqrt{\lambda_{n m}} b\right) f_{n m}}{\sqrt{a}\left[c J_{3 / 2-\gamma}\left(\delta_{n m}\right)\right]^{2} \Gamma\left(\frac{1}{2}-\beta\right) I_{1 / 2-\beta}\left(\sqrt{\lambda_{n m}} b\right)} .
\end{gathered}
$$

Substituting the values of coefficients $a_{0 m}, a_{n m}, b_{0 m}$ and $b_{n m}$ into (19), we find the formal solution of the Dezin problem in the form

$$
u(x, y, z)=\frac{2}{a} \sum_{n=0}^{\infty} \sum_{m=1}^{\infty}\left(\sin \frac{2 \pi n x}{a}+\cos \frac{2 \pi n x}{a}\right) \frac{z^{1 / 2-\gamma} J_{1 / 2-\gamma}\left(\delta_{n m} z / c\right)}{\left[c J_{3 / 2-\gamma}\left(\delta_{n m}\right)\right]^{2}} \omega_{n m}(y),
$$


where

$$
\begin{aligned}
\omega_{n m}(y)=\frac{y^{1 / 2-\beta} I_{1 / 2-\beta}\left(\sqrt{\lambda_{n m}} y\right)}{b^{1 / 2-\beta} I_{1 / 2-\beta}\left(\sqrt{\lambda_{n m}} b\right)}\left[g_{n m}-\bar{K}_{1 / 2-\beta}\left(\sqrt{\lambda_{n m}} b\right) f_{n m}\right]+ \\
+\bar{K}_{1 / 2-\beta}\left(\sqrt{\lambda_{n m}} y\right) f_{n m}
\end{aligned}
$$

where function $\bar{K}_{\nu}(x)[25]$ has the form: $\bar{K}_{\nu}(x)=2^{1-\nu} x^{\nu} K_{\nu}(x) / \Gamma(\nu), \bar{K}_{\nu}(0)=1 \quad(\nu>0)$.

Each member of this series satisfies all conditions of the Dezin problem. Let us note that when $\beta, \gamma<1 / 2$ the denominator of the coefficients of series (26) has no zeros. If we prove that series (26) and serieses $u_{x x},\left(u_{y y}+(2 \beta / y) u_{y}\right),\left(u_{z z}+(2 \gamma / z) u_{z}\right)$, obtained from it by differentiation converge absolutely and uniformly in corresponding domains then its sum is the solution of the Dezin problem. Moreover, we need the following lemmas.

Lemma 2. For any positive integers $n, m$ and $\forall y \in[0, b]$ the estimates

$$
\begin{gathered}
\left|\omega_{n m}(y)\right| \leqslant 2\left(\left|g_{n m}\right|+\left|f_{n m}\right|\right), \\
\left|y^{-2 \beta}\left[y^{2 \beta} \omega_{n m}^{\prime}(y)\right]^{\prime}\right| \leqslant 2 \lambda_{n m}\left(\left|g_{n m}\right|+\left|f_{n m}\right|\right)
\end{gathered}
$$

are valid.

Proof. Obviously, for any values of $\lambda_{n m}$ and $y \in[0, b]$ the inequality

$$
\left|\bar{K}_{1 / 2-\beta}\left(\sqrt{\lambda_{n m}} y\right)\right| \leqslant 1
$$

is valid. Since $y \in[0, b]$, and $I_{1 / 2-\beta}\left(\sqrt{\lambda_{n m}} y\right)$ is increasing function, then

$$
\frac{y^{1 / 2-\beta} I_{1 / 2-\beta}\left(\sqrt{\lambda_{n m}} y\right)}{b^{1 / 2-\beta} I_{1 / 2-\beta}\left(\sqrt{\lambda_{n m}} b\right)} \leqslant 1 .
$$

We obtain from (27) that

$$
y^{-2 \beta}\left[y^{2 \beta} \omega_{n m}^{\prime}(y)\right]^{\prime}=\lambda_{n m} \omega_{n m}(y) .
$$

If inequalities (30) and (31) are taken into account then estimates (28) and (29) immediately follow from (27) and (32). Lemma 2 is proved.

Lemma 3. The following estimates hold for $n \in N$ :

$$
\begin{gathered}
\left|\sin \frac{2 \pi n x}{a}+\cos \frac{2 \pi n x}{a}\right| \leqslant \sqrt{2}, \\
\left|\frac{d}{d x}\left(\sin \frac{2 \pi n x}{a}+\cos \frac{2 \pi n x}{a}\right)\right| \leqslant \sqrt{2} \mu_{n} .
\end{gathered}
$$

The validity of estimates (33)-(34) follows from the properties of trigonometric functions.

Lemma 4. For all natural numbers $n$ and $z \in[0, c]$ the following estimates hold for sufficiently large $m$,:

$$
\begin{gathered}
\left|Z_{n m}(z)\right| \leqslant C_{1}, \\
\left|z^{-2 \gamma}\left[z^{2 \gamma} Z_{n m}^{\prime}(z)\right]^{\prime}\right| \leqslant C_{1}\left(\delta_{n m} / c\right)^{2},
\end{gathered}
$$

where $C_{1}$ is some positive constant. 
Proof. It is obvious that $Z_{n m}(z) \in C[0, a]$. For sufficiently large $\xi$ the asymptotic formula holds [26]

$$
J_{\nu}(\xi) \approx\left(\frac{2}{\pi \xi}\right)^{1 / 2} \cos \left(\xi-\frac{\nu \pi}{2}-\frac{\pi}{4}\right) .
$$

Therefore, estimate (35) is true.

Using the first formula (17), we find from (15) that

$$
z^{2 \gamma} Z_{n m}^{\prime}(z)=\left(\delta_{n m} / c\right) z^{1 / 2+\gamma} J_{-1 / 2-\gamma}\left(\delta_{n m} z / c\right) .
$$

Let us take the first-order derivative of function (38) using formula (17). Then, multiplying it by $z^{-2 \gamma}$, we obtain

$$
z^{-2 \gamma}\left[z^{2 \gamma} Z^{\prime}{ }_{n m}(z)\right]^{\prime}=-\left(\delta_{n m} / c\right)^{2} z^{1 / 2-\gamma} J_{1 / 2-\gamma}\left(\delta_{n m} z / c\right)=-\left(\delta_{n m} / c\right)^{2} Z_{n m}(z) .
$$

Then validity of estimate (36) follows from (35).

Lemma 5. The following estimate holds for each fixed $n \in N$ and sufficiently large positive integer $m$ :

$$
J_{3 / 2-\gamma}^{2}\left(\delta_{n m}\right) \geqslant \frac{C_{2}}{\delta_{n m}}
$$

where $C_{2}$ is some positive constant.

Proof. Since $\delta_{n m}$ is a zero of function $J_{1 / 2-\gamma}(x)$ then relation

$$
\int_{0}^{c} z J_{1 / 2-\gamma}^{2}\left(\frac{\delta_{n m} z}{c}\right) d z=\frac{c^{2}}{2} J_{3 / 2-\gamma}^{2}\left(\delta_{n m}\right) .
$$

is true. It follows from this relation that

$$
J_{3 / 2-\gamma}^{2}\left(\delta_{n m}\right)=\frac{2}{c^{2}} \int_{0}^{c} z J_{1 / 2-\gamma}^{2}\left(\frac{\delta_{n m} z}{c}\right) d z=\frac{2}{\delta_{n m}^{2}} \int_{0}^{\delta_{n m}} \xi J_{1 / 2-\gamma}^{2}(\xi) d \xi .
$$

Taking into account asymptotic formula (37), there exists a sufficiently large number $c_{0}>0$ such that for $\xi>c_{0}$ equality

$$
\xi J_{1 / 2-\gamma}^{2}(\xi) \approx \frac{2}{\pi} \sin ^{2}\left(\xi+\frac{\gamma \pi}{2}\right) .
$$

is true. If we assume that $\delta_{n m}$ is a sufficiently large number and $\delta_{n m}>2\left(c_{0}+1\right)$ then

$$
\begin{array}{r}
\int_{0}^{\delta_{n m}} \xi J_{1 / 2-\gamma}^{2}(\xi) d \xi>\int_{c_{0}}^{\delta_{n m}} \xi J_{1 / 2-\gamma}^{2}(\xi) d \xi \geqslant \frac{2}{\pi} \int_{c_{0}}^{\delta_{n m}} \sin ^{2}\left(\xi+\frac{\gamma \pi}{2}\right) d \xi= \\
=\frac{1}{\pi} \delta_{n m}-\frac{1}{\pi}\left[c_{0}+\cos \left(\delta_{n m}+c_{0}+\gamma \pi\right) \sin \left(\delta_{n m}-c_{0}\right)\right] \geqslant \frac{1}{2 \pi} \delta_{n m} .
\end{array}
$$

Taking this into account, we obtain that estimate (39) follows from (40). Lemma 5 is proved.

Lemma 6. Let the following conditions be satisfied

$$
\begin{gathered}
\lim _{x \rightarrow 0} \frac{\partial^{k}}{\partial x^{k}} f_{j}(x, z)=\lim _{x \rightarrow a} \frac{\partial^{k}}{\partial x^{k}} f_{j}(x, z), \quad k=\overline{0,2}, \quad j=\overline{1,2}, \\
f_{j}(x, 0)=0, \quad f_{j}(x, c)=0, \quad f_{j z}(x, z) \in C([0, a] \times[0, c]), j=\overline{1,2}, \\
\lim _{z \rightarrow 0}\left[f_{j z z}(x, z)+\frac{2 \gamma}{z} f_{j z}(x, z)\right]=0, \lim _{z \rightarrow c}\left[f_{j z z}(x, z)+\frac{2 \gamma}{z} f_{j z}(x, z)\right]=0, \quad j=\overline{1,2},
\end{gathered}
$$




$$
\begin{gathered}
\frac{\partial}{\partial z}\left[f_{j z z}(x, z)+\frac{2 \gamma}{z} f_{j z}(x, z)\right] \in C([0, a] \times[0, c]), \quad j=\overline{1,2}, \\
\int_{0}^{c} \int_{0}^{a}\left|z^{-1 / 2-\gamma} \frac{\partial}{\partial z}\left\{z^{2 \gamma} \frac{\partial}{\partial z}\left[z^{-2 \gamma} \frac{\partial}{\partial z}\left(z^{2 \gamma} f_{j x x x z}(x, z)\right)\right]\right\}\right| d x d z<+\infty, \quad j=\overline{1,2} .
\end{gathered}
$$

Then, for large $n$ and $m$ we have estimates

$$
\left|f_{n m}\right| \leqslant \frac{C_{3}}{n^{3+\varepsilon_{7}} \delta_{n m}^{4+\varepsilon_{8}}}, \quad\left|g_{n m}\right| \leqslant \frac{C_{4}}{n^{3+\varepsilon_{7}} \delta_{n m}^{4+\varepsilon_{8}}},
$$

where $\varepsilon_{7}, \varepsilon_{8}, C_{3}, C_{4}$ are positive constants.

Proof. Using formulas (17), coefficients $f_{n m}$ are presented in the form

$$
f_{j n m}=\frac{a c}{2 \pi n \delta_{n m}} \int_{0}^{c} \int_{0}^{a} \frac{d}{d x}\left(\cos \frac{2 \pi n x}{a}-\sin \frac{2 \pi n x}{a}\right) \frac{d}{d z}\left[z^{1 / 2+\gamma} J_{-1 / 2-\gamma}\left(\frac{\delta_{n m} z}{c}\right)\right] f_{1}(x, z) d x d z .
$$

Taking into account conditions (41)-(44) at $j=1$ and applying the rule of integration by parts three times for variable $x$ and four times for variable $z$, we obtain

$$
\begin{aligned}
f_{n m}= & \frac{a^{3} c^{4}}{(2 \pi n)^{3} \delta_{n m}^{4}} \int_{0}^{c} \int_{0}^{a} \frac{\partial}{\partial z}\left\{z^{2 \gamma} \frac{\partial}{\partial z}\left[z^{-2 \gamma} \frac{\partial}{\partial z}\left(z^{2 \gamma} f_{1 x x x z}(x, z)\right)\right]\right\} \times \\
& \times\left(\sin \frac{2 \pi n x}{a}+\cos \frac{2 \pi n x}{a}\right) z^{1 / 2-\gamma} J_{1 / 2-\gamma}\left(\frac{\delta_{n m} z}{c}\right) d x d z .
\end{aligned}
$$

It is known [27] that if $f(x)$ is an absolutely integrable function on $[a, b]$ then equalities

$$
\lim _{n \rightarrow \infty} \int_{a}^{b} f(x) \cos n x d x=\lim _{n \rightarrow \infty} \int_{a}^{b} f(x) \sin n x d x=0 .
$$

are true. There is an analog of properties (48) [27]:

$$
\lim _{n \rightarrow \infty} \int_{0}^{1} x f(x) J_{p}\left(\lambda_{n} x\right) d x=0,
$$

here $f(x)$ is an absolutely integrable function on $[0,1]$, and $\lambda_{n}, n \in N$ are positive zeros of function $J_{p}(x)(p>-1)$ numbered in ascending order.

Since conditions (45) are satisfied then by virtue of (48)-(49) the following equality takes place

$$
\begin{aligned}
& \lim _{n, m \rightarrow \infty} \int_{0}^{c} \int_{0}^{a} \frac{\partial}{\partial z}\left\{z^{2 \gamma} \frac{\partial}{\partial z}\left[z^{-2 \gamma} \frac{\partial}{\partial z}\left(z^{2 \gamma} f_{1 x x x z}(x, z)\right)\right]\right\} \times \\
& \times\left(\sin \frac{2 \pi n x}{a}-\cos \frac{2 \pi n x}{a}\right) z^{1 / 2-\gamma} J_{1 / 2-\gamma}\left(\frac{\delta_{n m} z}{c}\right) d x d z=0 .
\end{aligned}
$$

Using (47) and the latter relation and assuming that $n$ and $m$ are sufficiently large, estimate (46) is obtained. The second estimate in (46) is proved similarly. Lemma 6 is proved.

Now we turn to the study of convergence of series. Differentiating (26), we obtain

$$
u_{x x}=\frac{2}{a} \sum_{n=0}^{\infty} \sum_{m=1}^{\infty}\left(\sin \frac{2 \pi n x}{a}+\cos \frac{2 \pi n x}{a}\right)^{\prime \prime} \frac{Z_{n m}(z) \omega_{n m}(y)}{\left[c J_{3 / 2-\gamma}\left(\delta_{n m}\right)\right]^{2}},
$$




$$
\begin{aligned}
& u_{y y}+\frac{2 \beta}{y} u_{y}=\frac{2}{a} \sum_{n=0}^{\infty} \sum_{m=1}^{\infty}\left(\sin \frac{2 \pi n x}{a}+\cos \frac{2 \pi n x}{a}\right) \frac{y^{-2 \beta}\left[y^{2 \beta} \omega^{\prime}{ }_{n m}(y)\right]^{\prime}}{\left[c J_{3 / 2-\gamma}\left(\delta_{n m}\right)\right]^{2}} Z_{n m}(z), \\
& u_{z z}+\frac{2 \gamma}{z} u_{z}=\frac{2}{a} \sum_{n=0}^{\infty} \sum_{m=1}^{\infty}\left(\sin \frac{2 \pi n x}{a}+\cos \frac{2 \pi n x}{a}\right) \frac{z^{-2 \gamma}\left[z^{2 \gamma} Z_{n m}^{\prime}(z)\right]^{\prime}}{\left[c J_{3 / 2-\gamma}\left(\delta_{n m}\right)\right]^{2}} \omega_{n m}(y) .
\end{aligned}
$$

Taking into account estimates (28), (33), (35) and (39), for sufficiently large natural numbers $n$ and $m$, series (26) for any $(x, y, z) \in[0, a] \times[0, \infty) \times[0, c]$ is majorized by the number series

$$
\sum_{n=0}^{\infty} \sum_{m=1}^{\infty} C_{5} \delta_{n m}\left(\left|g_{n m}\right|+\left|f_{n m}\right|\right)
$$

Series (50)-(52) on each compact $K \subset \Omega$ are majorized respectively by the following number series:

$$
\begin{gathered}
\sum_{n=1}^{\infty} \sum_{m=1}^{\infty} C_{6} n^{2} \delta_{n m}\left(\left|g_{n m}\right|+\left|f_{n m}\right|\right), \\
\sum_{n=0}^{\infty} \sum_{m=1}^{\infty} C_{7} \delta_{n m}\left(n^{2}+\delta_{n m}^{2}\right)\left(\left|g_{n m}\right|+\left|f_{n m}\right|\right), \\
\sum_{n=0}^{\infty} \sum_{m=1}^{\infty} C_{8} \delta_{n m}^{3}\left(\left|g_{n m}\right|+\left|f_{n m}\right|\right),
\end{gathered}
$$

where $C_{j}, j=\overline{5,8}$ are some positive constants.

According to [27, p. 276, formula (4.3)], for $\forall n \in N$ and sufficiently large positive integers $m$ inequality $\left(1 / \delta_{n m}\right) \leqslant(2 / m)$ is true.

Consequently, according to Lemma 6 , for sufficiently large $n$ and $m$ series (53)-(56) are estimated respectively by numerical series

$$
\sum_{n=1}^{\infty} \sum_{m=1}^{\infty} \frac{C_{9}}{n^{3+\varepsilon_{7}} m^{3+\varepsilon_{8}}}, \sum_{n=1}^{\infty} \sum_{m=1}^{\infty} \frac{C_{10}}{n^{1+\varepsilon_{7}} m^{3+\varepsilon_{8}}}, \sum_{n=1}^{\infty} \sum_{m=1}^{\infty} \frac{\left(n^{2}+m^{2}\right) C_{11}}{n^{3+\varepsilon_{7}} m^{3+\varepsilon_{8}}}, \sum_{n=1}^{\infty} \sum_{m=1}^{\infty} \frac{C_{12}}{n^{3+\varepsilon_{7}} m^{1+\varepsilon_{8}}},
$$

where $C_{j}, j=\overline{9,12}$ are some positive constants.

It is not difficult to establish that series (57) converge. Therefore, series (53)-(56) also converge. Then, according to the Weierstrass criterion, series (26) converges absolutely and uniformly in $\bar{\Omega}$, and series (50)-(52) converges on each compact $K \subset \Omega$. Therefore, function $u(x, y, z)$ defined by $(26)$ satisfies all conditions of the Dezin problem.

Thus, the following theorem is proved.

Theorem 2. Let function $f_{j}(x, z), j=\overline{1,2}$ satisfies conditions (41)-(45). Then a solution of the Dezin problem exists, it is unique, and it is determined by formula (26).

\section{References}

[1] A.A.Dezin, Operators involving a first derivative with respect to time and nonlocal boundary conditions, Math. USSR-Izv., 1(1967), no. 1, 57-79.

[2] A.M.Nakhushev, Equations of Mathematical Biology, Moscow, Visshaya shkola, 1995 (in Russian). 
[3] A.A.Guetter, A free boundary problem in plasma containment, SIAM J. Appl. Math., 49(1989), 99-115.

[4] C.V.Pao, Reaction diffusion equations with nonlocal boundary and nonlocal initial conditions, Journal of Mathematical Analysis and Applications, 195(1995), no. 3, 702-718. DOI: $10.1006 /$ jmaa.1995.1384

[5] E.Obolashvili, Nonlocal problems for some partial differential equations, Applicable Analysis, 45(1992), 269-280. DOI: 10.1080/00036819208840101

[6] J.I.Diaz, J.M.Rakotoson, On a nonlocal stationary free boundary problem arising in the confinement of a plasma in a Stellarator geometry, Archive for Rational Mechanics and Analysis, 134(1996), 53-95. https://doi.org/10.1007/BF00376255

[7] F.I.Frankl, Flowing profiles by a stream of subsonic velocity with a supersonic zone ending with a direct surge of seal, PMM, 20(1956), no. 2, 196-202.

[8] N.I.Ionkin, Solving one regional problem of thermal conductivity theory with a non-classical edge condition, Differ. equations, 13(1977), no. 2, 294-304. zbmath.org/0349.35040

[9] N.I.Ionkin, On the sustainability of one problem theory of thermal conductivity with a nonclassical edge condition, Differ. equations, 15(1979), no. 7, 1279-1283.

zbmath.org/0418.35053

[10] N.I.Ionkin, E.I.Moiseyev, About the task for the equation of thermal conductivity with twopoint edge conditions, Differ. equations, 15(1979), no. 7, 1284-1295.

zbmath.org/0415.35032

[11] M.E.Lerner, O.A.Repin, On Frankl'-type problems for some elliptic equations with degeneration of various types, Differ. equations, 35(1999), no. 8, 1098-1104. mi.mathnet.ru/de9976

[12] M.E.Lerner, O.A.Repin, Nonlocal boundary value problems in a vertical half-strip for a generalized axisymmetric Helmholtz Equation, Differ. equations, 37(2001), no. 11, 1562-1564. mi.mathnet.ru/de10495

[13] E.I.Moiseev, On the solution of a nonlocal boundary value problem by the spectral method, Differ. equations, 35(1999), no. 8, 1105-1112. mi.mathnet.ru/de9977

[14] E.I.Moiseev, Solvability of a Nonlocal Boundary Value Problem, Differ. equations, 37(2001), no. 11, 1643-1646. https://doi.org/10.1023/A:1017937403853

[15] Yu.K.Sabitova, Nonlocal initial-boundary-value problems for a degenerate hyperbolic equation, Russian Math. (Iz. VUZ), 53(2009), no. 12, 41-49. mi.mathnet.ru/ivm6024

[16] A.A.Abashkin, Vestn. Samar. Gos. Tekhn. Univ., Ser. Fiz.-Mat. Nauki (J. Samara State Tech. Univ., Ser. Phys. Math. Sci.), 36(2014), no. 3, 7-20 (in Russian). https://doi.org/10.14498/vsgtu1328

[17] A.M.Nakhushev, Offset problems for partial differential equations, Moscow, Nauka, 2006 (in Russian).

[18] Z.A.Nakhusheva, Nonlocal boundary value problems for basic and mixed types of differential equations, Nalchik, 2011 (in Russian). 
[19] Z.A.Nakhusheva, Differ. equations, 45(2009), no. 8, 1199-2003.

DOI: $10.1134 /$ S0012266109080151

[20] K.B.Sabitov, V.A.Novikova, Russian Math. (Iz. VUZ), 60(2016), no. 6, 52-62. DOI: 10.3103/S1066369X16060074

[21] K.B.Sabitov, Differ. equations, 55(2019), no. 10, 1384-1389.

DOI: $10.1134 /$ S0012266119100136

[22] A.N.Tikhonov, A.A.Samarskiy, Equations of mathematical physics, Moscow, Nauka, 1972 (in Russian).

[23] M.A.Naimark, Linear differential operators, Moscow, Nauka, 1969 (in Russian).

[24] G.N.Watson, Theory of Bessel functions, Moscow, Part. 1, Izd. IL., 1949 (in Russian).

[25] M.B.Kapilevich, On one equation of mixed elliptic-hyperbolic type, Matematicheskii Sbornik., 30:72(1952), 11-38 (in Russian).

[26] N.N.Lebedev, Special functions and their applications, Moscow, Fizmatlit, 1963 (in Russian).

[27] G.P.Tolstov, Fourier series, Moscow, Nauka, 1980 (in Russian).

\title{
Нелокальная задача для трехмерного эллиптического уравнения с сингулярными коэффициентами в прямоугольном параллелепипеде
}

Камолиддин Т. Каримов

Ферганский государственный университет Фергана, Узбекистан

\begin{abstract}
Аннотация. Исследована нелокальная задача для эллиптического уравнения с двумя сингулярными коэффициентами в прямоугольном параллелепипеде. Доказательство единственности решения и его построение проведены спектральным методом с использованием разложения в ряд Фурье и Фурье-Бесселя. При некоторых условиях относительно параметров и заданных функций доказана равномерная сходимость построенного ряда.
\end{abstract}

Ключевые слова: уравнения эллиптического типа, нелокальная задача, сингулярный коэффициент, спектральный метод, параллелепипед. 